\title{
Biobank attributes associated with higher patient participation: a randomized study
}

\author{
Angèle Gayet-Ageron ${ }^{\star, 1}$, Sandrine Rudaz ${ }^{1}$ and Thomas Perneger ${ }^{1}$
}

The objectives of the study were to assess patients' intent to participate in a hospital-based biobank and to explore the factors associated with higher participation. A 23-item questionnaire was developed to survey a random sample of patients in a Swiss university hospital. Two vignettes describing hypothetical biobanks were incorporated in the survey and patients were asked whether they would agree to participate. Three factors were randomly manipulated in each vignette using a factorial design: cancer-oriented research vs general consent, one vs several reviews of the patient's chart, and genetic vs blood protein analyses (first vignette); blood sample vs oral swabbing, local vs international project, and a follow-up visit vs no visit (second vignette). Of the 1140 respondents, 73.6 and $69.6 \%$, respectively, agreed to participate in the biobank. Biospecimen collection via oral swabbing, single chart review, and no follow-up were associated with higher participation. Participation was also higher among younger patients, Europeans, patients who had a positive opinion on research, and blood/organ donors. Biobanking was supported by a majority of patients, especially if biospecimens were collected through non-invasive techniques or if data collection was done once. The scope of consent, the scale of the project, or the tests performed on biospecimens did not influence participation.

European Journal of Human Genetics (2017) 25, 31-36; doi:10.1038/ejhg.2016.132; published online 5 October 2016

\section{INTRODUCTION}

Genetic epidemiology relies increasingly on the availability of biobanks. ${ }^{1}$ However, biobanks face several challenges related to the need to protect research subjects and to provide in return a societal benefit attributable to the research findings. ${ }^{2,3}$ Patients are key actors in the development of a hospital-based biobank and their active support is an essential element for its success. Many biobanks obtain specimens from patients at the point of care, such as at the time of hospital admission. Although participation rates are typically high, the elements that make participation in the biobank attractive to patients are only partially understood. ${ }^{4}$

Several factors associated with the intent of patients to participate in a biobank have been studied previously. First, the scope of consent can influence participation. One-time consent compared to dynamic or tiered consent, ${ }^{5,6}$ or general consent compared to specific consent ${ }^{7}$ were associated with an increased intended participation. Patients expressed the possibility to withdraw consent even after inclusion in the biobank as important even if in reality this is a right and not an option. ${ }^{5}$ Second, a one-time data collection from the medical chart and the absence of follow-up over time was associated with increased participation. ${ }^{8}$ The type of biobank sample has not been specifically assessed in the literature; especially no study has compared the willingness to participate in a biobank that uses left-over blood after routine clinical visits compared with the ad hoc collection of blood samples or oral swabs. Because the latter procedures entail an additional burden to the patient, this factor may influence patient participation. Finally, individual characteristics can influence participation in a biobank, such as older age, being married or being a blood donor. ${ }^{9}$
This study aimed to assess the effect of several biobank attributes on intended participation among hospitalized patients. We performed an experimental survey among patients discharged from a university hospital in Switzerland based on two hypothetical vignettes presenting a biobank project. Each vignette tested three experimental factors that were randomly attributed to potential participants in order to control for the effect of various confounders.

\section{MATERIALS AND METHODS}

Study design, setting and participants

We designed a cross-sectional study in a random sample of patients hospitalized between 1 March and 31 March 2014, at the University of Geneva Hospitals of Geneva, a 2000-bed public teaching hospital located in Geneva, Switzerland, with $>48000$ admissions per year, representing $>670000$ hospitalization days. Participants were adults that had been hospitalized and who were identified through the administrative database. Exclusion criteria were residence outside Switzerland or lack of a home address (in-transit patients). A random sample of 2600 patients was selected and a survey package including an information letter, a questionnaire, and a stamped return envelope was sent by post mail 8-12 weeks after discharge. Two reminders were sent during the next 2 months.

The study protocol was submitted to the institutional review board, which exempted it from formal review because it carried minimal risk.

\section{Questionnaire and clinical research vignettes}

We developed a 23-item questionnaire that included three parts. The first part contained seven items assessing the respondents' opinion about participation in research. The second part presented four clinical vignettes, including two about a biobank project within the hospital (Supplementary Appendix 1A and B). Each clinical vignette tested three binary factors, which were randomly attributed using a factorial design, thus yielding 8 versions (A to $\mathrm{H}$ ) of the

\footnotetext{
${ }^{1}$ Division of Clinical Epidemiology, Department of Health and Community Medicine, University of Geneva Hospitals and Faculty of Medicine, 6 Rue Gabrielle Perret-Gentil, Geneva 14, Switzerland

${ }^{*}$ Correspondence: A Gayet-Ageron, Division of Clinical Epidemiology, Department of Health and Community Medicine, University Hospitals of Geneva and Faculty of Medicine, 6 Rue Gabrielle Perret-Gentil, Geneva 14, 1211 Switzerland. Tel: +41 22372 9027; Fax: +41 22 372 9035; E-mail: angele.gayet-ageron@hcuge.ch

Received 4 May 2016; revised 11 July 2016; accepted 5 August 2016; published online 5 October 2016
} 
survey (Supplementary Appendix 2). The third part recorded participant characteristics (see independent variables below). Initial versions of the vignettes were pre-tested by six persons to assess the readability and ensure that they were easily understood by French speakers.

\section{Primary outcome}

At the end of each vignette, the respondents were asked to give their position about their hypothetical participation (primary outcome) on a 5-point Likert scale: (1) 'I would have certainly refused'; (2) 'I would have probably refused'; (3) 'I would have hesitated'; (4) 'I would have probably agreed'; and (5) 'I would have certainly agreed'.

Independent variables associated with the willingness to participate For each vignette, the three experimental factors were the main predictors tested to explain the willingness to participate (Supplementary Appendix 2). In the first vignette, the factors tested were the scope of consent (general consent $v s$ cancer-oriented research), one $v s$ several reviews of the patient's chart, and the type of analyses done on the biospecimens (genetic vs blood protein analyses). In the second vignette, we tested the method used for collecting biospecimens (blood sample $v s$ oral swabbing), patient follow-up visit (yes vs. no), and the scale of the biobank project (local vs international). We investigated also the following patient characteristics and their past behavior or opinion toward research: age; gender; country of birth; level of education; childbearing and marital status; self-rated health status; opinion on the utility of clinical research at the hospital and genetic research in particular; past participation in clinical research; and if they were blood or organ donors.

\section{Sample size estimation}

We anticipated that $70 \%$ of respondents would have a positive opinion toward a hospital-based biobank. ${ }^{10}$ To obtain a $95 \%$ confidence interval (CI) of $\pm 2.5 \%$ around $70 \%, 1300$ patients were required. We expected that patient participation would be $50 \%$, leading to a total number of 2600 patients or 325 per vignette version. This study size allowed detection of a $7 \%$ difference in positive opinion (70 vs 77\%) between two categories of experimental factors at a power level of $80 \%$.

\section{Statistical analysis}

The two vignettes were analyzed separately. For each vignette, we first estimated the intent to participate by grouping 'I would have certainly agreed' with 'I would have probably agreed' and estimated the 95\% CI using the exact binomial method. We then assessed the two primary outcomes using the original 5-point Likert scale (ordinal format). We used an ordered logistic regression model for each vignette to estimate the association between the likelihood of participation as the dependent variable and the three dichotomous experimental factors as the independent variables. In a second step, we forced all the experimental factors into the models and added pre-specified individual characteristics. Age groups were categorized as $<40,40-59,60-74$ and $\geqslant 75$ years. Education levels were grouped as follows: 'elementary school and apprenticeship' $v s$ 'secondary school' $v s$ 'professional school and university' as the reference. Patient self-rated health status was ordered from 'poor' to 'excellent', then dichotomized as 'excellent/very good/good' vs 'fair/poor'. Patient opinion on clinical research was ordered from 'very negative' to 'very positive'. Patient opinion on genetic research was grouped in 'positive opinion' $v s$ 'negative opinion' or 'no opinion'. All analyses were performed using Stata version intercooled 14 (StataCorp., College Station, TX, USA). Statistical significance was defined as $P<0.05$ (two-sided).

\section{RESULTS}

\section{patient characteristics}

Of 2600 randomly selected patients, 1140 (43.8\%) returned the completed questionnaires (Table 1); 1118 of 1140 (98.1\%) responded to at least one vignette about the biobank and were included in the current analysis. Reasons for non-participation were refusal $(n=32)$, death $(n=22)$ and failure to return the questionnaire $(n=1406)$. More than $50 \%$ of the respondents were women (Table 1). Half of all respondents were born in Switzerland, 55.9\% had completed elementary school or an apprenticeship, 53.6\% were married, and $78.5 \%$ had at least one child. The mean age was 60 years. Respondents considered their health as excellent or very good in $23.2 \%$ of cases and $32.0 \%$ had been hospitalized in the last 6 months. One-quarter of respondents had participated in at least one clinical study in the past (Table 2). Respondents had a favorable global opinion about clinical research and genetic research in particular.

\section{Experimental factors associated with willingness to participate}

The first vignette was completed by 1108 (42.6\%) patients, the second by $1102(42.4 \%)$, and 1092 patients completed both (42.0\%). The percentage of answers from a certain refusal to a certain agreement increased gradually from $5.2 \%$ to $43.6 \%$ in the first vignette (Figure 1a), and from 7.2 to $38.4 \%$ in the second vignette (Figure 1b). In the first vignette, 816 (73.6\%; 95\% CI: 70.9-76.2)

\section{Table 1 Responders' characteristics}

\begin{tabular}{|c|c|}
\hline Variables & Respondents $\left(\mathrm{n}=1118^{\mathrm{a}}\right)$ \\
\hline Female gender, $n(\%)$ & $614(55.6)$ \\
\hline Mean age (SD, median) & $60.0( \pm 19.4,63)$ \\
\hline \multicolumn{2}{|l|}{ Categories of age (years), n (\%) } \\
\hline$<40$ & $209(19.8)$ \\
\hline $40-59$ & $267(25.3)$ \\
\hline $60-74$ & $287(27.1)$ \\
\hline$\geqslant 75$ & $294(27.8)$ \\
\hline \multicolumn{2}{|l|}{ Country of birth, n (\%) } \\
\hline Switzerland & $573(52.0)$ \\
\hline Other European countries & $360(32.7)$ \\
\hline Other countries & $169(15.3)$ \\
\hline \multicolumn{2}{|l|}{ Level of education, $\mathrm{n}(\%)$} \\
\hline Elementary school & $266(24.2)$ \\
\hline Apprenticeship & $348(31.7)$ \\
\hline Secondary school & 119 (10.9) \\
\hline Professional school & $141(12.8)$ \\
\hline University & $224(20.4)$ \\
\hline \multicolumn{2}{|l|}{ Marital status, n (\%) } \\
\hline Married & $590(53.6)$ \\
\hline Single, divorced, separated, widowed & $510(46.4)$ \\
\hline \multicolumn{2}{|l|}{ Has children, n (\%) } \\
\hline Yes & $863(78.5)$ \\
\hline No & $236(21.5)$ \\
\hline \multicolumn{2}{|l|}{ Self-rated health status, n (\%) } \\
\hline Excellent & $66(6.0)$ \\
\hline Very good & $189(17.2)$ \\
\hline Good & $517(47.2)$ \\
\hline Fair & $250(22.8)$ \\
\hline Poor & $74(6.8)$ \\
\hline \multicolumn{2}{|l|}{ Blood donor, n (\%) } \\
\hline Yes & $361(33.0)$ \\
\hline Tried & $99(9.0)$ \\
\hline No & $636(58.0)$ \\
\hline \multicolumn{2}{|l|}{ Organ donor card, n (\%) } \\
\hline Yes & $200(18.2)$ \\
\hline Not yet & $145(13.2)$ \\
\hline No & $752(68.6)$ \\
\hline Hospital stay in the last 6 months, $n(\%)$ & $338(32.0)$ \\
\hline
\end{tabular}

\footnotetext{
aSome data had missing values, \% calculated on available data; missing data were excluded.
} 
would have certainly or probably agreed to participate compared with 768 (69.7\%; 95\% CI: 66.9-72.4) in the second vignette. There were no differences between the eight groups of patients randomly allocated to the different versions of the vignettes for all covariates tested or in terms of the response rate (data not shown, available upon request).

Among the six experimental factors randomly manipulated in the vignettes, three were significantly associated with willingness to participate (Table 3). In the first vignette, a single review of the patient's chart (compared with several reviews) was independently associated with a greater likelihood to participate. The type of biological analyses performed on biospecimens or the scope of consent presented to patients were not associated with participation. In the second vignette, a higher willingness to participate was found if biospecimens were obtained by oral swabbing compared with a blood sample, or if the patients did not require a follow-up visit. Of note, willingness to participate was not associated with the scale of the project.

Individual predictors associated with willingness to participate After adjustment for individual-level predictors, the associations between willingness to participate and the three experimental factors tested in each clinical vignette remained unchanged (Table 4). In both vignettes, we found similar independent associations between willingness to participate and the following individual predictors: younger respondents; individuals born in Switzerland or other European countries (vignette 2); those who had a positive opinion of clinical research or had participated in a clinical study in the past or had a favorable opinion on genetic research; blood donors and potential organ donors.

\section{Table 2 Responders' opinion toward research}

\begin{tabular}{|c|c|}
\hline Variables & $\begin{array}{l}\text { Respondents } \\
\left(\mathrm{n}=1118^{\mathrm{a}}\right)\end{array}$ \\
\hline Participation to clinical studies during the last hospital stay, $n(\%)$ & $275(25.0)$ \\
\hline Past participations to clinical studies, $n(\%)$ & $274(24.9)$ \\
\hline \multicolumn{2}{|l|}{ Research is an important mission of a university hospital, $\mathrm{n}$ (\%) } \\
\hline Very important & $937(84.5)$ \\
\hline Rather important & $154(13.9)$ \\
\hline Not important & $18(1.6)$ \\
\hline \multicolumn{2}{|l|}{$\begin{array}{l}\text { Is it justified to ask patients to contribute to producing knowledge } \\
\text { that will be useful to other persons? n (\%) }\end{array}$} \\
\hline Definitively justified & $876(79.3)$ \\
\hline Partially justified & $199(18.0)$ \\
\hline Definitively unjustified & $29(2.6)$ \\
\hline \multicolumn{2}{|l|}{ What is your opinion about clinical research among patients? n (\%) } \\
\hline Very positive & $448(40.6)$ \\
\hline Rather positive & $483(43.7)$ \\
\hline Neutral & $150(13.6)$ \\
\hline Rather negative & $17(1.5)$ \\
\hline Very negative & $6(0.5)$ \\
\hline \multicolumn{2}{|l|}{ Opinion of genetic research, $\mathrm{n}(\%)$} \\
\hline Favorable & $765(69.3)$ \\
\hline Non expressed & $303(27.5)$ \\
\hline Unfavorable & $36(3.3)$ \\
\hline
\end{tabular}

aSome data had missing values, \% calculated on available data; missing data were excluded.

\section{DISCUSSION}

In this experimental study conducted in a sample of patients in a Swiss university hospital, the willingness to participate in a hypothetical research biobank was high with $\sim 70 \%$ of positive answers. Intended participation was increased if the biospecimens were to be collected through oral swabbing ( $v s$ blood sample), if the patient was not followed over time, or if his/her medical record was consulted only once. We did not observe any influence of the scope of consent (general $v s$ cancer-oriented), the use of genetic tests ( $v s$ blood protein analysis) on biospecimens, or the scale of the project (local vs international). Patient characteristics associated with willingness to participate in a biobank were a younger age ( $<75 v s \geq 75$ years) and Swiss or European nationality ( $v s$ nationality from other countries). Having a positive opinion on clinical research or genetic research, having participated to clinical studies in the past, or being a blood or organ donor were also independent predictors of intended participation in the biobank.

Attitudes of patients toward genetic research and biobanking ${ }^{10-13}$ are often more favorable than the attitudes of the general population. ${ }^{14-17}$ This patient bias may be explained by patients' belief that research findings would potentially benefit their own health. ${ }^{10,18-21}$ However, willingness to participate in a hypothetical biobank may be lower than the actual participation because some determinants of behavior, such as altruism, trust or sense of duty are less implied in the decision process in a fictional situation. ${ }^{5,20-23}$

Some of our results confirmed previous reports, such as an increase in willingness to participate if data were collected once, ${ }^{8}$
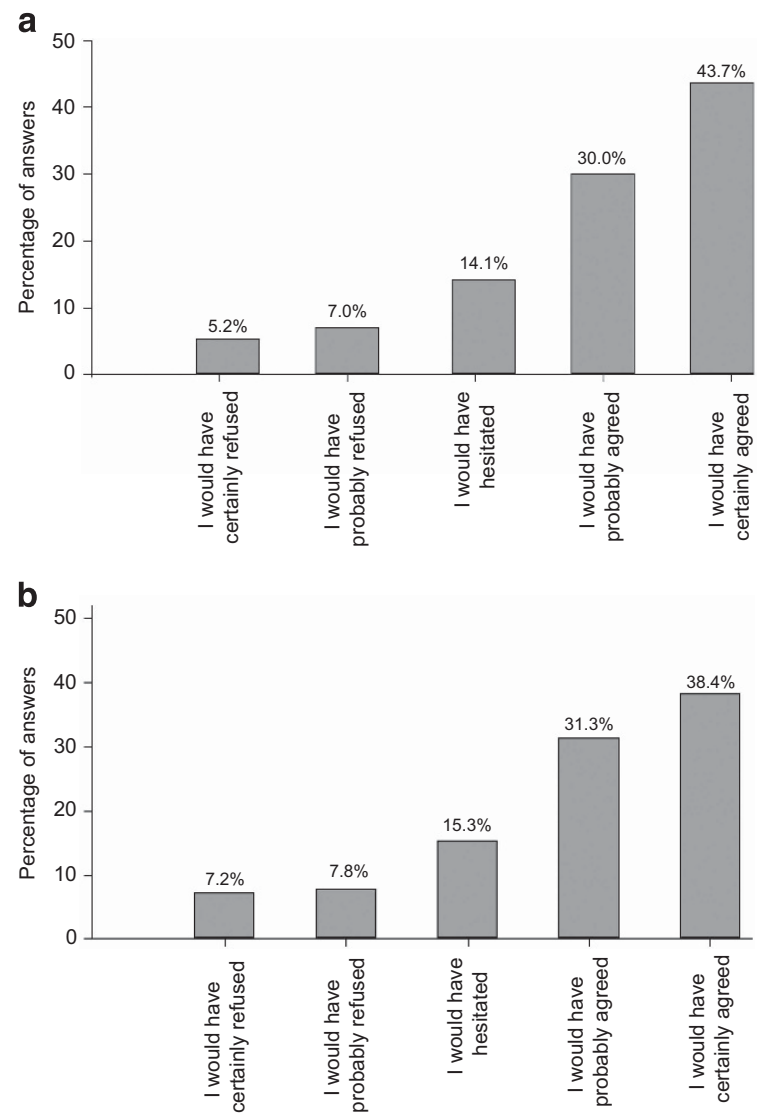

Figure 1 Distribution of the percentage of answers from certain refusal to certain approval in the first vignette (a) and second vignette (b). 
Table 3 Independent associations of six experimental factors with willingness to participate in a biobank project

\begin{tabular}{|c|c|c|c|}
\hline \multirow[b]{2}{*}{ Experimental factors assessed in the two clinical vignettes } & \multicolumn{3}{|c|}{ Willingness to participate } \\
\hline & Odds ratio & $95 \% \mathrm{Cl}$ & P-value \\
\hline \multicolumn{4}{|l|}{ Vignette 1} \\
\hline Cancer-oriented consent (vs general consent) & 0.90 & $0.72-1.12$ & 0.35 \\
\hline Single review of the patient's chart or individual health data (vs several reviews) & 1.35 & $1.09-1.68$ & 0.007 \\
\hline Genetic analyses on the biospecimens (vs blood protein analysis) & 1.11 & $0.89-1.38$ & 0.35 \\
\hline \multicolumn{4}{|l|}{ Vignette 2} \\
\hline Oral swabbing (vs blood sample) & 1.35 & $1.09-1.67$ & 0.007 \\
\hline No follow-up of patients (vs follow-up) & 1.54 & $1.24-1.91$ & $<0.001$ \\
\hline Local project (vs international) & 0.88 & $0.71-1.10$ & 0.27 \\
\hline
\end{tabular}

Abbreviation: $\mathrm{Cl}$, confidence interval.

a Obtained by ordinal logistic regression model. Willingness to participate was rated on a 5-point Likert scale.

Table 4 Multiple-ordinal logistic regression models per clinical vignette

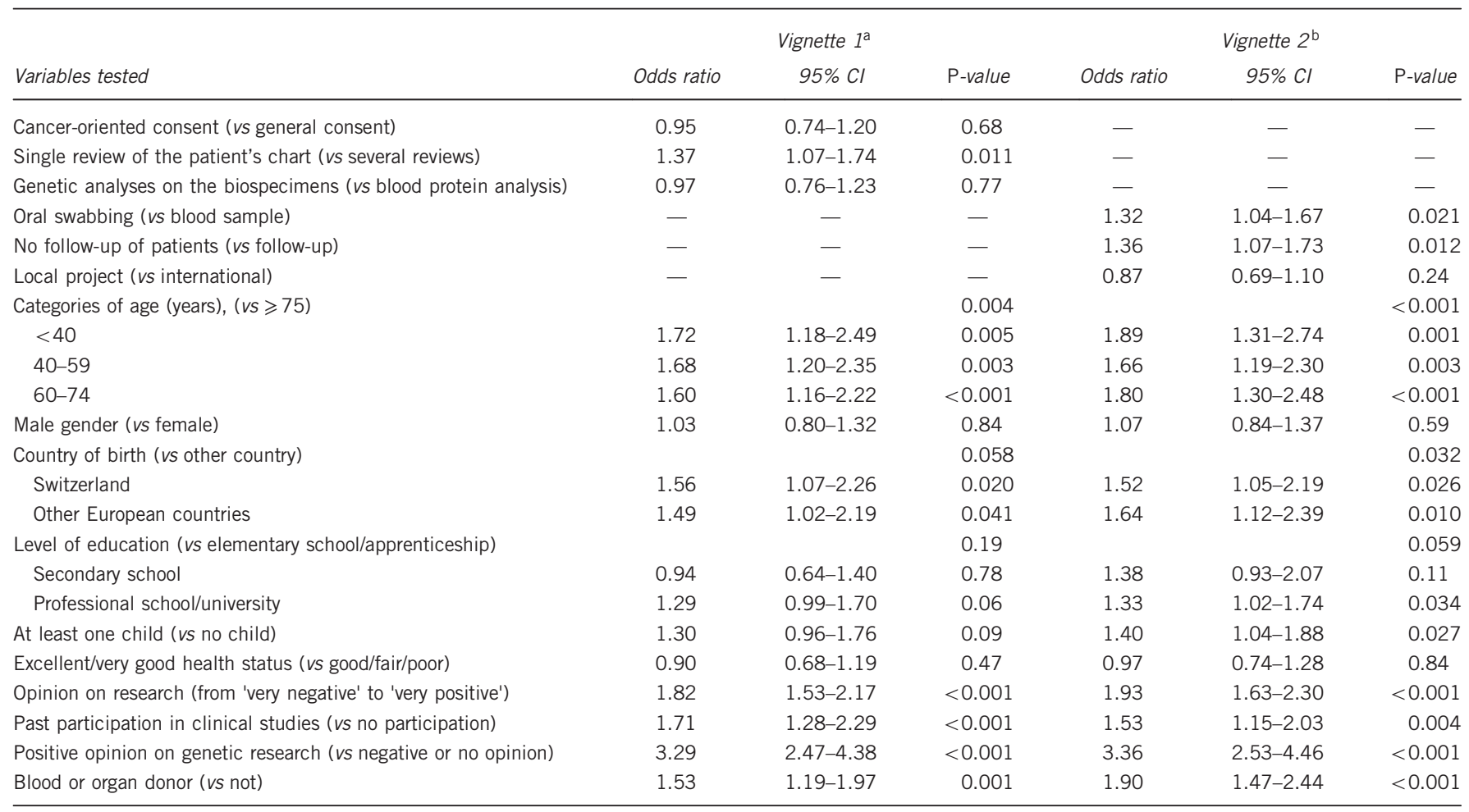

Abbreviation: $\mathrm{Cl}$, confidence interval.

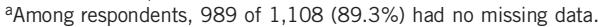

bAmong respondents, 992 of $1,102(90.0 \%)$ had no missing data.

if patients were not re-contacted ${ }^{13}$ or if the biospecimens were collected through oral swabbing and not as a blood sample. ${ }^{13}$ In contrast to previous findings, ${ }^{6,24}$ we did not find any association between participation and the scope of consent. A similar lack of association was also recently reported in a survey conducted in the USA. ${ }^{25}$ Two possible explanations can be proposed. First, the scope of consent may not be of concern to patients because they trust the research project investigators. ${ }^{20}$ Alternatively, patients do not understand the implications of broad consent compared with disease-oriented consent or study-specific consent and this leads them to ignore this factor during the decision process. ${ }^{26}$ In general, when broad consent is sought, its implications should be clearly stated in the information sheet distributed to potential participants. In particular, patients should understand that they will not be informed each time a new research project makes use of their biospecimens.

We expected that patients would feel less comfortable with genetic analyses than with other types of research, which would reduce their willingness to participate in a genetic biobank, but no such effect was found. We presume that this absence of association may be due to a lack of knowledge about the potential risks related to confidentiality in genetic research or the 
consequences related to an incidental genetic variant discovery. ${ }^{23}$ Similar to a US trial where $53 \%$ of respondents were willing to donate a blood sample because of a specific interest in genetic research, ${ }^{16}$ the majority of participants $(\sim 70 \%)$ in our study were favorable to genetic research. Finally, the local $v s$. international scale of the biobank did not influence the respondents' willingness to participate.

The association between patient age and the willingness to participate in a biobank has been investigated on several occasions. In some studies, intended participation increased with age, $9,27,28$ while the opposite was true in others. ${ }^{8,18,29}$ This suggests that age in itself may be less important than other respondent characteristics that may vary with age, such as expectations or attitudes, or that the effect of age is sensitive to the type of biobank proposed. Similarly, the culture and attitudes toward research probably explain the effect of patient nationality on participation in the biobank. Finally, previous participation in a medical research study or the expression of a favorable opinion regarding research, particularly genetic research, are naturally associated with participation in a biobank as the attitudes, preferences and values required are essentially the same. Several studies have reported similar findings. ${ }^{10,18,30,31}$ Being a blood donor or having an organ donation card are associated with a positive attitude toward genetic research $^{9,31,32}$ and these characteristics indicate also that a person is comfortable with making part of his/her body available for the benefit of others.

The main strength of this study is that we targeted patients who are directly concerned by the development of hospital-based biobanks. The experimental design of the study allowed to control for the effect of confounding on the association between the six factors tested and the intended participation of patients in biobanking. However, the limited participation in the survey may have caused a selection bias with respondents more motivated by the topic or more favorable to biobanks than nonparticipants. ${ }^{28}$ Other limitations of our study deserve mention. First, we only tested factors assessing the logistics of a biobank. Because we were limited in the number of experimental factors we did not assess other potentially important factors, such as the return of individual genetic test results. Patient attitudes toward the return of incidental genomic results vary, and can be influenced by disease severity, disease treatability, carrier status, life expectancy, likelihood of response to treatment, and cost of the test. ${ }^{33}$ It was also demonstrated that return of incidental findings was generally better accepted by the general population than by patients. ${ }^{34,35}$ Similarly, the willingness to participate in a hospitalbased biobank may be influenced by the possibility of sharing data between several data sets and also of disseminating the results through online publications. ${ }^{36}$ Finally, factors related to culture and the local socio-political system, such as altruism, or the relative weight of individual versus societal interests were not assessed here. Surveyed populations from Northern European countries are often more motivated to participate in a biobank research than Southern European countries; ${ }^{37}$ Switzerland is at the lower range of motivated countries. For these reasons, our results may not be generalizable to other cultures.

In conclusion, our results suggest that patients are more willing to participate in biobanks if the procedures are simple and non-invasive. Intent to participate in biobanking was not influenced by the scope of consent, the type of analyses done on the biospecimens, or the scale of the biobank project.

\section{CONFLICT OF INTEREST}

The authors declare no conflict of interest.

\section{ACKNOWLEDGEMENTS}

We thank Rosemary Sudan for editorial assistance.

1 Khoury MJ, Millikan R, Little J, Gwinn M: The emergence of epidemiology in the genomics age. Int J Epidemiol 2004; 33: 936-944.

2 Ashburn TT, Wilson SK, Eisenstein BI: Human tissue research in the genomic era of medicine: balancing individual and societal interests. Arch Intern Med 2000; 160: 3377-3384.

3 Hofmann B: Broadening consent-and diluting ethics? J Med Ethics 2009; 35: 125-129.

4 Mooser V, Currat C: The Lausanne Institutional Biobank: a new resource to catalyse research in personalised medicine and pharmaceutical sciences. Swiss Med Wkly 2014; 144: w14033.

5 Master Z, Claudio JO, Rachul C, Wang JC, Minden MD, Caulfield T: Cancer patient perceptions on the ethical and legal issues related to biobanking. BMC Med Genomics 2013; 6: 8

6 Platt J, Bollinger J, Dvoskin R, Kardia SL, Kaufman D: Public preferences regarding informed consent models for participation in population-based genomic research. Genet Med 2014; 16: 11-18.

7 Ruiz-Canela M, Valle-Mansilla JI, Sulmasy DP: Researchers' preferences and attitudes on ethical aspects of genomics research: a comparative study between the USA and Spain. J Med Ethics 2009; 35: 251-257.

8 Ahram M, Othman A, Shahrouri M, Mustafa E: Factors influencing public participation in biobanking. Eur J Hum Genet 2014; 22: 445-451.

9 Kerath SM, Klein G, Kern M et al: Beliefs and attitudes towards participating in genetic research-a population based cross-sectional study. BMC Public Health 2013; 13: 114.

10 Rahm AK, Wrenn M, Carroll NM, Feigelson HS: Biobanking for research: a survey of patient population attitudes and understanding. J Community Genet 2013; 4: 445-450.

11 Bryant RJ, Harrison RF, Start RD et al: Ownership and uses of human tissue: what are the opinions of surgical in-patients? J Clin Pathol 2008; 61: 322-326.

12 Pillai U, Phillips K, Wilkins G et al: Factors that may influence the willingness of cancer patients to consent for biobanking. Biopreserv Biobank 2014; 12: 409-414.

13 McVeigh TP, Sweeney KJ, Kerin MJ, Gallagher DJ: A qualitative analysis of the attitudes of Irish patients towards participation in genetic-based research. Ir J Med SCl 23, 2015.

14 Kettis-Lindblad A, Ring L, Viberth E, Hansson MG: Genetic research and donation of tissue samples to biobanks. What do potential sample donors in the Swedish general public think? Eur J Public Health 2006; 16: 433-440.

15 Nilstun T, Hermeren G: Human tissue samples and ethics-attitudes of the general public in Sweden to biobank research. Med Health Care Philos 2006; 9: 81-86.

16 Wang SS, Fridinger F, Sheedy KM, Khoury MJ: Public attitudes regarding the donation and storage of blood specimens for genetic research. Community Genet 2001; 4: 18-26.

17 Wong ML, Chia KS, Yam WM, Teodoro GR, Lau KW: Willingness to donate blood samples for genetic research: a survey from a community in Singapore. Clin Gene 2004; 65: 45-51.

18 Trauth JM, Musa D, Siminoff L, Jewell IK, Ricci E: Public attitudes regarding willingness to participate in medical research studies. J Health Soc Policy 2000; 12 $23-43$.

19 Klima J, Fitzgerald-Butt SM, Kelleher KJ et al: Understanding of informed consent by parents of children enrolled in a genetic biobank. Genet Med 2014; 16 : $141-148$.

20 McDonald JA, Vadaparampil S, Bowen D et al: Intentions to donate to a biobank in a national sample of African Americans. Public Health Genomics 2014; 17: $173-182$.

21 Bryant J, Sanson-Fisher R, Fradgley E, Regan T, Hobden B, Ackland SP: Oncology patients overwhelmingly support tissue banking. BMC Cancer 2015; 15: 413.

22 Johnsson L, Helgesson G, Rafnar $T$ et al: Hypothetical and factual willingness to participate in biobank research. Eur J Hum Genet 2010; 18: 1261-1264.

23 Ormond KE, Cirino AL, Helenowski IB, Chisholm RL, Wolf WA: Assessing the understanding of biobank participants. Am J Med Genet 2009; 149: 188-198.

24 Simon CM, L'Heureux J, Murray JC et al: Active choice but not too active: public perspectives on biobank consent models. Genet Med 2011; 13: 821-831.

25 Ewing AT, Erby LA, Bollinger J, Tetteyfio E, Ricks-Santi LJ, Kaufman D: Demographic differences in willingness to provide broad and narrow consent for biobank research. Biopreserv Biobank 2015; 13: 98-106.

26 Tomlinson T, De Vries R, Ryan K, Kim HM, Lehpamer N, Kim SY: Moral concerns and the willingness to donate to a research biobank. JAMA 2015; 313: 417-419.

27 Lee $\mathrm{Cl}$, Bassett LW, Leng $\mathrm{M}$ et al: Patients' willingness to participate in a breast cancer biobank at screening mammogram. Breast Cancer Res Treat 2012; 136: 899-906.

28 Ridgeway JL, Han LC, Olson JE et al: Potential bias in the bank: what distinguishes refusers, nonresponders and participants in a clinic-based biobank? Public Health Genomics 2013; 16: 118-126. 
29 Banks E, Herbert N, Mather T, Rogers K, Jorm L: Characteristics of Australian cohort study participants who do and do not take up an additional invitation to join a long-term biobank: The 45 and Up Study. BMC Res Notes 2012; 5: 655.

30 Porteri C, Pasqualetti P, Togni E, Parker M: Public's attitudes on participation in a biobank for research: an Italian survey. BMC Med Ethics 2014; 15: 81

31 Al-Jumah MA, Abolfotouh MA: Public perception and attitude of saudis toward organ and tissue donation. Biopreserv Biobank 2011; 9: 21-27.

32 Rice MS, Custer BS, Hindes DA et al: Genetic research in the blood bank: acceptability to Northern California donors. Transfusion 2010; 50: 1951-1958.

33 Bennette CS, Trinidad SB, Fullerton SM et al: Return of incidental findings in genomic medicine: measuring what patients value-development of an instrument to measure preferences for information from next-generation testing (IMPRINT). Genet Med 2013; 15: 873-881.
34 Meulenkamp TM, Gevers SK, Bovenberg JA, Koppelman GH, van Hylckama Vlieg A, Smets EM: Communication of biobanks' research results: what do (potential) participants want? Am J Med Genet 2010; 152: 2482-2492.

35 Bollinger JM, Scott J, Dvoskin R, Kaufman D: Public preferences regarding the return of individual genetic research results: findings from a qualitative focus group study. Genet Med 2012; 14: 451-457.

36 Carey DJ, Fetterolf SN, Davis FD et al: The Geisinger MyCode community health initiative: an electronic health record-linked biobank for precision medicine research. Genet Med 2016; 18: 906-913.

37 Gaskell G, Gottweis H, Starkbaum J et al: Publics and biobanks: Pan-European diversity and the challenge of responsible innovation. Eur J Hum Genet 2013; 21: 14-20.

Supplementary Information accompanies this paper on European Journal of Human Genetics website (http://www.nature.com/ejhg) 